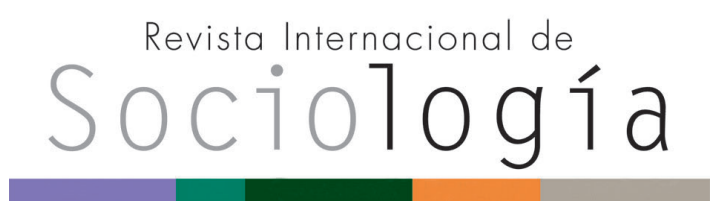

Revista Internacional de Sociología RIS

vol. 74 (3), e038, julio-septiembre, 2016, ISSN-L:0034-9712

doi: http://dx.doi.org/10.3989/ris.2016.74.3.038

\section{AMARTYA SEN FRENTE AL ESPEJO SOCIAL DE LA LIBERTAD. Límites al enfoque de las capacidades individuales}

\author{
Manuel Antonio Jiménez Castillo \\ El Colegio de la Frontera Norte, México. \\ antoniojcastillo@colef.mx
}

Cómo citar este artículo / Citation: Jiménez Castillo, A. 2016. "Amartya Sen frente al espejo social de la libertad. Límites al enfoque de las capacidades individuales". Revista Internacional de Sociología 74 (3): e038. doi: http://dx.doi. org/10.3989/ris.2016.74.3.038

\section{Resumen}

En este trabajo se ilustran algunos aspectos relativos al enfoque de las capacidades desarrollado por el economista y filósofo Amartya Sen. Particularmente se examina cómo el concepto de libertad ("de oportunidad") se revela imposibilitado para atenerse empírica y normativamente a la noción de libertad como capacidad. A partir de tal contradicción categorial se presentan una serie de conceptos inéditos que, vinculados al carácter más social de la libertad, permitan librarnos de tal desajuste. Las conclusiones de este trabajo van enfocadas hacia la enfatización del entorno social como estado donde, lejos de su reconocida influencia sobre las capacidades individuales, atesora elementos potencialmente capacitadores.
\end{abstract}

\section{Palabras Clave}

Capacidad social; Contra-capacidad; Libertad; Metacapacidad.

\section{AMARTYA SEN FROM SOCIAL MIRROR OF FREEDOM. Limits to Individual Capabilities Approach}

Copyright: @ 2016 CSIC. Este es un artículo de acceso abierto distribuido bajo los términos de la licencia Creative Commons Attribution (CC BY) España 3.0.

Recibido: 26/12/2014. Aceptado: 01/07/2015. Publicado on line: 22/08/2016

\section{Abstract}

This paper illustrates somehow aspect of the capabilities approach developed by the economist and philosopher Amartya Sen. Particularly it examines how the concept of freedom (as "opportunity") reveals unable to empirically and normatively adjust to the notion of freedom as capability. From such categorical contradiction is introduced a set of unprecedented concepts that, gathered to the largest social dimension of freedom, allow releasing from such mismatch. Conclusions are focused on the emphasis of social environment as state that far from its recognized influence on individual capabilities has potentially enablers' elements.

\section{KEYWORDS}

Counter-Capability; Freedom; Meta-Capability; Social Capability. 


\section{GÉNESIS DEL ASUNTO}

La teoría de las capacidades (capabilities approach) desarrollada durante más de tres lustros por el economista y filósofo indio Amartya Sen ha recibido recientemente una muy abultada aunque asimétrica atención en círculos académicos y civiles. Esta disimilitud basculada hacia lo menos científico del asunto ha alimentado, en ocasiones, una inflación de textos cuyo pretexto atiende a unas formas desprovistas de sustancia. Reorientar tales costumbres exige un juicio introspectivo sobre los fundamentos mismos que originaron tal enfoque y que negocian hoy sobre nuevos horizontes epistémicos. Introducirse en esta tarea será el propósito de este trabajo.

El enfoque de Amartya Sen se encuentra originariamente vertebrado en relación con el concepto de capacidad ("libertad como capacidad") desde el cual, se pretende, a partir de una reflexión hermenéutica de la diversidad, construir un enfoque de justicia social donde el punto de inflexión sea la calidad de vida desde aquello que el sujeto es capaz de lograr realmente (Sen 1998; 2009; 2013).Aunque Sen (1999) habla desde un primer momento de libertades reales como "una expansión de las oportunidades reales con las que los individuos puedan asumir aquel tipo de vida que consideran más deseable", la variable focal que se presta a la consecución de tal estado de libertad es una que el mismo califica "de oportunidad". Ello avoca desde un inicio a un reduccionismo analítico incontestable pues toma como real de la libertad tan solo su vinculación positiva de oportunidad "libre para" (1985a). A pesar de que ello pudiera mostrarse irrelevante a propósito de la implícita dimensión positiva que indefectiblemente acompaña al concepto de libertad real "expansión de las oportunidades reales [...]", no lo es de ningún modo. Ello se debe particularmente a que el mero ejercicio analítico de la libertad "de oportunidad" supone en sí mismo una actitud contradictoria donde lo teorizado responde a un desplazamiento de aquellos fenómenos que hipotecarán su posibilidad fáctica (Deneulin 2009; 2014).

Sen es en todo momento consciente de esta apreciación al reconocer cómo la teoría de corte republicano o neorromano (libertad como no-dominación) captura más eficazmente aquellas interferencias de lo que lo hace la libertad "de oportunidad", donde "la capacidad para controlar y determinar las propias acciones" son trascendidas de la mera voluntad individual (Sen 1999; 2009). Sin embargo, la interpretación que de tal hecho conviene el economista de Cambridge pareciera estar más orientado a las deficiencias inevitables de cualquier teoría que se enfrente a lo real que a la imposibilidad operativa del propio enfoque. De este modo, la teoría seniana de capacidad se embarca analíticamente en un proceso de autojustificación desde un marco empírico de acción (libertad para funcionar realmente) que no es el que le viene dado de su constitución teórica (libertad "de oportunidad"), pero que se le supone con tal de no tener que asumir las implicaciones analíticas de un enfoque inaca- bado. Hablar de libertades reales desde la estrecha dimensión de la libertad "de oportunidad" sin incluir su otra dimensión "de procesos" "libre de" impedimentos y/o coacciones (Sen 1988), supone una marginalización de aquellos elementos que determinan el grado normativo y operativo de la libertad misma que defiende (Deneulin 2011; Agee y Crocker 2012).

En este trabajo sostendremos cómo desde la libertad "de oportunidad" es empírica y normativamente implausible una expansión asintótica de las libertades reales de los individuos que, siendo en última instancia el fundamento último del enfoque, deviene de una definitiva correspondencia entre la libertad "de oportunidad y procesos". En consecuencia trabajaremos en una serie de novedosos conceptos que nos permita readaptar la libertad "de oportunidad" a los cambios derivados de la incorporación de la libertad "de procesos" como covariable focal del enfoque. Si bien, no será la libertad "de procesos" en toda su dimensión abarcable en este trabajo. Tal hecho requeriría de la identificación de los innumerables fenómenos que se revelan en tanto que impedimento al libre ejercicio de la libertad. A tal fin, restringiremos la cuestión al concepto de entorno social examinado por Deneulin (2006; 2011), entendiéndolo como el conjunto de estructuras políticas, económicas y sociales que, depositarias de la libertad "de procesos", afectan profusamente a la "de oportunidad" y, con esta, a las libertades reales. Tomaremos como referente teórico lo denominado como "dimensión social de la libertad" ("de procesos"), siendo desde una equitativa distribución de recursos determinante para identificar aquellos funcionamientos (functionings) -aquello que una persona consigue hacer o estar- cuya posibilidad reposa en las condiciones materiales y estados resultantes del entorno social y no en la mera voluntad individual de acción (libertad "de oportunidad") (Deneulin 2011; 2014). Así, frente al concepto de capacidades individuales con el que Sen denota no más que al conjunto de funcionamientos posibles (libertad para funcionar), se impone otro de naturaleza más universalista ("capacidades absolutas"1).Un concepto cuya significación descansa en la libertad real como síntesis siempre inconclusa y dada a partir de una conformidad entre las capacidades individuales como fruto de la libertad "de oportunidad", y las "capacidades sociales" y "metacapacidades". Estas últimas como resultado de la incorporación de la libertad "de procesos" (en su dimensión social) a la base informacional de las capacidades. Un concepto absoluto de capacidad con el que se pretende no más que adaptar el juicio normativo que ya mantiene su autor para las capacidades individuales, pero que nos permita reunir bajo un marco perfeccionado aquellos otros fenómenos que afectan a la determinación fáctica y normativa de la libertad real. 
El artículo se divide siguiendo tres objetivos específicos: en primer lugar, se exponen las limitaciones empíricas de la libertad "de oportunidad" en el marco seniano. En segundo lugar, se examina cómo la dimensión social libertad ("de procesos"), objetivada desde el concepto de entorno social y mediatizada a su vez a un estado de equidad distributiva, remedia el irrealismo empírico y normativo de la libertad "de oportunidad". A tal fin, se incorporan una serie de conceptos-"capacidades sociales", "contracapacidades" y "metacapacidades"-que nos faciliten resolver de alguna forma el abandono analítico de la libertad "de proceso" y sus implicaciones en la generación de las capacidades. Finalmente, sintetizaremos los aspectos y las contribuciones más relevantes de este trabajo.

\section{Amartya Sen y la libertad: estado CONTROVERTIDO DE LA CUESTIÓN}

Aunque el concepto de libertad utilizado por Amartya Sen es uno "de oportunidad", las pretensiones de su autor son en todo momento vincularlas al concepto de igualdad -en un sentido de equidad rawlsaniana-, cuando afirma "una teoría de la justicia tiene que estar viva tanto para la rectitud de los procesos implicados como para la equidad y la eficacia de las oportunidades (...)" (Sen 2009:326). Sen, que hasta entonces ha sido muy crítico con Rawls y con el enfoque de las necesidades básicas ante la incapacidad de salvar el pluralismo de formas (Sen 1973b; 2000) -libertades sociales en su sentido más amplio-, se ve por primera vez frente a un conflicto de tipo universalista. El economista indio, consciente de lo que queda fuera de la dimensión "de oportunidad", sostiene que sin un relativo sentido distributivo sería fáctica y normativamente implausible cualquier teoría de justicia social, pues más allá de lo dado, las condiciones materiales determinan empíricamente las oportunidades reales (Sen, op. cit.). Esta autoadvertencia recogida en la crítica al óptimo paretiano como el "espíritu del César" que "viene caliente del infierno" (Sen 1986:34) queda restringida a un ámbito meramente intencional -en ningún momento la traslada a su enfoque- sometiendo cualquier éxito de la teoría a la habilidad de su autor para hacer frente a unas críticas crecientes (Nussbaum 2002; 2011; Deneulin 2011; Steward 2013).

Para superar el conflicto, que deviene de apartar de lo teórico lo exigido desde la razón práctica, el autor de las capacidades realiza un giro dialéctico esperado. Defendiendo un ideal de vida aristotélico a partir de un claro dualismo kantiano, Sen (2002) diluye el concepto de libertad "de oportunidad" en un principio de "universalidad abstracta", suspendiendo así la operatividad del enfoque al principio de "validez discursiva". La cuestión distributiva es reconocida tan solo a partir de un principio de discusión más elevado -en este caso las oportunidades para funcionar (Sen 1984; 1985b)-. Esta contradicción de naturaleza performativa por cuanto in actu intenta negar cualquier principio distributivo, si bien lo hace siempre utilizándolo. La igualdad, como reconocimiento de lo que "de procesos" tiene la libertad real, solo lo es a partir de unos niveles abstractos elevados pues cualquier vinculación empírica la revelaría del todo implausible. De este modo, la libertad seniana ("de oportunidad") consigue trascender a todas sus objetivaciones, aunque no puede existir sin ella. La libertad "de oportunidades" es en todo momento una libertad restringida hacia lo que el individuo puede realmente hacer y en nada hacia lo que se lo impide -libertad "de procesos"-, ya que aunque en ocasiones lo incluye, no son en ningún caso la misma cosa ${ }^{2}$. Desde la estrecha frontera de la libertad "de oportunidad", se impone el problema de fondo de la inasumible "facticidad" de las oportunidades reales a no ser que se acepte a priori el irrealismo del enfoque; en otros términos, suponer como lógica una estructura factual que en sí es irreal. Así, centrándose el enfoque en aquello que el individuo es capaz de hacer, termina inevitablemente dejando de lado aquellas dinámicas de poder que, entre otras y como sostiene Cejudo (2007:18) "forman parte del proceso histórico de consecución de bienestar social".

La aseveración de Cejudo no es un aliciente para la teoría de las capacidades y sí una crítica en toda regla, ya que si el enfoque pretende formular un juicio efectivo sobre la calidad de vida, no parece razonable que lo haga sin tener en cuenta aquellos fenómenos que la delimitan. Autores como Van Parijs (1996), Giri (2000) Van Staveren (2001) y Deneulin (2006; 2009; 2011) han corroborado, -siguiendo una línea de corte claramente individualista en los tres primeros- la pronunciada devaluación que supone para la teoría seniana la negación analítica de sus implicaciones sociales. "Una sociedad cuyos miembros son realmente libres requiere que la libertad se componga de seguridad, propiedad de sí y oportunidad, y no solo de esta última" (Van Parijs 1996:42). Para ellos, tomar como variable focal la libertad "de oportunidad" supondría una pérdida irremediable del sentido descriptivo de la libertad como capacidad.

Esto nos traslada al hecho fundamental que marcará el déficit de toda su estructura teórica y que, a la par, fundamenta el sentido de las siguientes páginas. Si la calidad de vida se compone de la libertad para poder funcionar (capabilities) a partir de los distintas oportunidades disponibles, los logros realmente alcanzados serán, de acuerdo con Sen (1984), el indicador más efectivo de justicia social. Empero, si los logros no son resultados de la mera libertad "de oportunidad" sino que, como iremos demostrando a la luz de lo ya advertido, responden en igual medida a la dimensión social de la libertad ("de procesos"), no podrán entenderse desde la exclusiva referencia indicativa de bienestar. Siendo esto tal que así, nos exigirá una reinterpretación de los logros -ya sean estos en forma de 
recursos o estados- donde más allá de su naturaleza condicionante de buena vida (Rawls 1971; Streeten 1996; Cortina 2002) o resultado mismo de las distintas formas para funcionar (Sen 1984;2006;2009),"manifiestan ser simultáneamente generadores de capacidades". Pero no de capacidades como libertades de las cosas para sí, sino como disposiciones con las que contribuir al desarrollo de los funcionamientos individuales. No es solo, tal y como apunta el enfoque de las capacidades, lo que los individuos como agentes hacen o son con los bienes poseídos ${ }^{3}$, sino lo que los bienes pueden hacer por ellos más allá de satisfacer, entre otras, sus necesidades básicas.

De esta interpretación se revela cómo los bienes trascienden el sentido más seniano de deducirlos desde aquello que los individuos pueden llegar a hacer con ellos a la hora de expandir sus libertades reales. Una interpretación que, superando el individualismo ético que arrastra todo el marco categórico seniano, se hace a nuestro juicio imprescindible para revelar específicamente de qué modo los distintos estados sociales, entendidos desde la relación dialéctica entre libertad "de oportunidad" y "procesos", determinan el cómo se ordenan y disponen las distintas formas que permiten funcionar realmente.

\section{LA DIMENSIÓN SOCIAL DE LA LIBERTAD COMO CAPACIDAD}

\section{Capacidades sociales: raíces conceptuales}

Que Sen defina "la libertad" como 'capacidad' desde una dimensión ético-individualista "de oportunidad" implica negar una parte importante de lo que la libertad personal es, y que Berlín (2002:15) solo la rescata parcialmente cuando la define como aquello que ocurre "sin coerción, sin presiones, sin verse engullido por un vasto sistema y en el derecho a oponerse, a ser impopular, a defender convicciones propias simplemente porque son tus convicciones". Esta fórmula restrictiva de entender la libertad "de procesos" como mero "obstáculo para" estaría ocultando, como atenderemos en las siguientes líneas, un rol más profundo como generador de capacidades.

A pesar de que la teoría seniana advierte el por qué en ciertas ocasiones una embarazada necesita más recursos para tener las mismas capacidades que un hombre, no es capaz de explicar de qué manera la sociedad dificulta en términos de privación de oportunidades las decisiones que una embarazada pueda llegar a tomar realmente (Sen 2009). De estas palabras se advierte la precariedad con la que Sen asimila el entorno social (libertad "de procesos"), donde aquellas relaciones interpersonales -conjunciones entre estados individuales y sociales en el sentido más amplio-, quedan negadas por un modelo tan invisible a la dimensión social de la libertad ("de oportunidad") como a aquellos fenómenos que determinan su posibilidad.
Una de las implicaciones más relevantes que derivan de equipararla libertad "de oportunidad" a la real, es la restricción a nivel operativo del concepto de capacidad al de funcionamiento ${ }^{4}$. Aunque Sen deja clara tal distinción a lo largo de su obra, solo se hace expresa en términos analíticos y nunca empíricos (Sen 1997; 1999). Ilustremos esta cuestión desde el siguiente ejemplo: ser capaz de leer y escribir entendido en términos de funcionamiento primitivohacer $x$ sin importar que otras posibles alternativas existen-es, por un lado, independiente de cualquier entorno social especificado -puedo leer y escribir en cualquier entorno posible en términos de posibilidad lógica-; y por otro, dependiente del mismo entorno social dado, pues las posibilidades para funcionar realmente vendrán empíricamente mediatizadas. Un individuo capaz de leer puede sentirse tentado a no hacerlo no porque lo decide libremente sino porque se encuentra sometido a un entorno social que le es desfavorable -represión cultural de la China maoísta o la Camboya de Pol Pot-. Desde la teoría seniana ambas situaciones -leer o no hacerlo- serían interpretadas como óptimas de bienestar, pues el enfoque es incapaz de advertir aquellos estados cuya posibilidad empírica deriva de las restricciones sociales del entorno (libertad "de procesos").

Este aspecto cobra una mayor relevancia cuando examinamos los funcionamientos refinados. Entendidos como la posibilidad de "escoger hacer $\mathrm{x}$ cuando otras alternativas están disponibles", es utilizado por Sen para subrayar la relevancia dela libertad sobre el logro alcanzado. No obstante, el concepto de funcionamiento refinado no repara ni en la naturaleza de las alternativas presentes ni en cómo estas determinan la posibilidad e intensidad para funcionar. De este modo, el autor acaba trasladando al plano lógico lo que corresponde a la dimensión estrictamente empírica. Acto que no es en sí sinónimo de garantía, pues en las ciencias empíricas puede ser lógico -funcionar desde alternativas teóricamente plausibles- aquello que es empíricamente implausible -hacerlo desde un entorno social desfavorable. Sen (2009), que no niega lo implausible de tal hecho, no le bastará para que incorpore aquello que permita juzgar su posibilidad. Desde esta imposibilidad factual fundamentará toda su estructura analítica.

Llegados a este punto, aparece en escena el con-

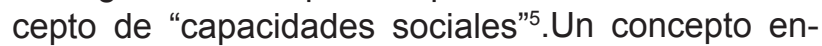
tendido como conjunto de posibilidades que desde lo social (entorno) permiten establecer las oportunidades reales con las que funcionar. Tal estado determina las oportunidades reales de los individuos desde el condicionamiento de su libertad "de oportunidad" a partir del desarrollo de la dimensión social de la libertad ("de procesos") (Deneulin 2006; 2014). De este modo, las "capacidades sociales" no se agotan como barreras que superar a fin de expandir las libertades reales sino que, más allá de ello, se constituyen como 
fundamento para su posibilidad fáctica. Si bien aquellas no se presentan como sustitutivas de las capacidades individuales y sí como complementarias. Su sentido último queda especificado en la proyección que de lo exógeno -entorno social capacitador- genera el propio individuo derivado de esa misma condición de exogeneidad. Una condición (entorno social) que reúne para sí aquellos medios con los que poder funcionar y que es propio e independiente de cualquier estado individual de funcionamiento, aunque no pueda serlo para sí. Si bien el entorno social es un ente capacitador, su resolución en cuanto que pura manifestación empírica solo podrá procurarse desde su encuentro con el individuo, donde en última instancia el funcionamiento se consuma.

\section{Injerencias en la dimensión social de la libertad: las contracapacidades}

Cuando Sen diseña la base informacional de su teoría desde las cuatro categorías básicas (libertad, bienestar, agencia y logro) no proporciona todas las variables con las que hacer de la libertad una posibilidad fáctica. Aunque los desarrollos desde el campo de la libertad y logro de bienestar han sido mucho más elaborados que los que tienen que ver con los logros (agency goals) y libertades de agencia (agency freedom) ${ }^{6}$ (Cejudo 2007), el hostigamiento de evaluarlos "desde" el sujeto y no "con" el sujeto, ha dificultado el uso de formulaciones analíticas alternativas. Cuando tomamos las variables de agencia y libertad de agencia, entendidas como el grado más elevado de libertad (Sen 1985a), examinarlas desde el individualismo ético de la libertad "de procesos" supone un "conflicto fatal" que termina derivando en severos desajustes conceptuales. Algunos de estos desajustes tienen que ver con las "capacidades sociales" en un sentido positivo. Otros, en cambio, sin menoscabo de lo anterior, lo hacen desde una manifestación negativa. Ilustremos esta cuestión comenzando con un ejemplo.

Supongamos que una persona decide contraer matrimonio en un entorno sometido a una arraigada base religiosa. Sería lógico preguntarse si la decisión de llevarlo a cabo viene motivada por su entera voluntad de satisfacer su bienestar personal, o si ocurre desde la influencia social en la que se encuentra inmersa. En este sentido, e indistintamente para ambos casos, podríamos hablar de éxito en cuanto a logro de bienestar, si bien, existirían serias dudas en relación a la libertad con la que es alcanzado. Imaginemos que el mismo individuo bajo idénticas condiciones culturales decide tajantemente no esposarse. Su capacidad como agente libre para decidir cómo actuar en pos de cualquiera objetivos y valores que considere importantes (libertad de agencia), se verá condicionada por el hecho de que la comunidad reconozca ese aspecto de su identidad, y que tenga, a su vez, un impacto efectivo en la forma de vida de los que le rodean. Mientras que desde una interpretación seniana podría entenderse que el individuo puede potencialmente actuar con libertad para esposarse (objetivo de bienestar) la realidad nos revelaría una restricción de tal posibilidad.

Para articular tal imposibilidades necesario incorporar al marco de "capacidades sociales" una nueva dimensión que nos permita identificar aquellas formas de darse el entorno social que supondrían, en caso de manifestarse, una restricción explícita a la libertad de bienestar y agencia. A tal fin hemos diseñado el concepto de "contracapacidades". Tal concepto que asume en términos negativos el marco de las capacidades sociales, no puede entenderse bajo un sentido de capacitación "negativa" como "descapacitación" pues las capacidades cuando son, lo son siempre para funcionar positivamente incluso cuando tal funcionamiento implique su negación como un no funcionamiento. Siguiendo con este razonamiento, las "contracapacidades" apuntarían entonces a aquellas formas de darse el entorno social que determinan negativamente el modoen el que se manifiesta la libertad (de agencia o bienestar) para funcionar. Este estado de negatividad como "contracapacidad" nos encamina necesariamente hacia la irrupción en el seno del enfoque seniano de un conflicto de naturaleza normativa entre la variable focal (libertad "de oportunidad") y el propio sentido de justicia (libertad como capacidad).Veamos esta cuestión con un poco de más detenimiento.

La relevancia del concepto de "contracapacidad" deriva en última instancia de la posibilidad para identificar aquellos elementos que irrumpidos desde el entorno social, restringen la libertad para funcionar. El abandono de esta cuestión por parte del marco seniano emana del hecho por el cual, la libertad para funcionar nunca se agota en sí misma. La libertad es consustancial al sujeto independientemente del grado de libertad con el que funcionar. Es por ello que cobra especial relevancia entender cuáles son aquellos escenarios (entorno social) donde la libertad interviene. Una relevancia que se vincula a la identificación de tales escenarios, y si desde ellos, el individuo experimenta un incremento real en sus funcionamientos. Si bien, ello solo es posible trascendiendo la propia manifestación fáctica de la libertad ("de oportunidad"). Este aspecto es de gran importancia ya que si asumimos la libertad, tal y como la presenta Sen, alcanzar grados de libertad en sí mismo no puede ser condición suficiente de bienestar, pues incluso en condiciones de perentoria esclavitud siempre se manifiestan alternativas que permiten funcionar. Esto nos transporta a un aspecto notable que Sen nunca llega a asumir y que deriva en un desdoblamiento de la propia concepción seniana de libertad, donde una positivización de la libertad-crecimiento asintótico de la libertad para funcionar- se emancipa de su sombra normativa. De este modo, más libertad ya no es sinónimo estricto de mejor libertad, o en otras palabras, la expansión de la libertad para funcionar deja de ser mi- 
mética expresión normativa de un perfeccionamiento en la calidad de vida. La significación ética dada a partir de ese mejoramiento positivo de libertades se libera para revelarse conflictivamente. Al soslayar esta distinción Sen termina irremediablemente confundiendo -voluntaria o involuntariamente- la expansión de las libertades reales como variable focal con la expansión de las libertades "normativamente deseables" . Siendo esto tal que así, surge una cuestión sucesiva y decisiva expresable en los siguientes términos: ¿por qué Sen no atiende a esta cuestión con implicaciones tan definitivas para el éxito de su teoría?

La respuesta podría ir vinculada a la supuesta percepción del logro como fuente intrínseca de normatividad. Sen sostiene desde un principio que el logro no es en sí suficiente para juzgar una vida como deseable, prestándose necesario valorar la libertad con la que este ha sido alcanzado (Sen 1982; 1998; 1999). Aunque Sen acierta, solo lo hace parcialmente, pues elude juzgar normativamente la supuesta importancia del logro en la libertad alcanzada. El logro adquiere una relevancia irreductible no solo para valorar los objetivos de bienestar y agencia (Sen 1984; 1985b; 2003), sino también la libertad con la que estos son alcanzados. De forma que si el logro solo puede ser completamente asimilado desde la libertad con el que es logrado, "la libertad para funcionar solo puede ser completamente asimilada desde el logro alcanzado". Tan relevante se presenta considerar la libertad para valorar el logro (Sen 1999), como el logro para valorar la libertad. Esta relación dialéctica que inevitablemente escapa al individualismo ético de Sen no es para nada sorprendente si tenemos en cuenta el restringido sentido de libertad ("de oportunidad") que vertebra su teoría. Ante tales circunstancias, Sen no tiene más remedio que fijar como criterio normativo de su teoría la libertad en un sentido positivo-incremento cuantitativo de las libertades-a riesgo de tener que asumir explícitamente la inoperatividad normativa de su enfoque.

\section{La seguridad humana como fundamento de libertad: el concepto de metacapacidad}

A esta altura del análisis, vamos a centrar la cuestión en el examen de unas formas particulares de darse el entorno social que son en sí favorables al desarrollo de los funcionamientos pero inasumibles desde los conceptos de "capacidades sociales" y "contracapacidades". A tal fin, incorporamos al análisis el concepto de "metacapacidad". La "metacapacidad", no nace en sí como un modo particular de funcionamiento, y sí como conjunto de estados específicos que lo permiten desde una particular forma de darse el entorno. Del estudio de esta particularidad y de sus implicaciones sobre los funcionamientos nos ocuparemos a continuación.

La "metacapacidad", entendida como la capacidad para estar capacitado, va a encontraren estas líneas una gran asociación analítica con el concep- to más evolucionado de seguridad humana como ausencia de vulnerabilidad (Otaga 2003), es decir, en la mitigación de aquellos estados que limitarían el proceso expansivo de la libertad real o efectiva. Vincular el entorno social al concepto de seguridad humana nos va a enfrentar ante situaciones hasta ahora desatendidas. Un entorno social proclive al desenvolvimiento de las capacidades individuales no debe focalizarse en el exclusivo bienestar personal si no esa condición de desatender aquellos conflictos potenciales entre objetivos de agencia (lograr lo valorable) y bienestar. Esta cuestión rebosa de significación pues se inmiscuye directamente en el grado normativo de la libertad, enlazándolo con las "capacidades sociales". Veámoslo más claramente con otro ejemplo tomado por Sen;

\footnotetext{
"Ante un conflicto militar una persona puede sentirse tentada a no abandonar el territorio a pesar de la consecuente reducción de bienestar personal que implica actuar de esta manera. Aunque decide tomar la decisión de permanecer aun teniendo alternativas, no la cambia por el simple hecho de que tal circunstancia se encuentra presente" (1998:283).
}

Atendiendo al texto, incluso existiendo funcionamientos alternativos estos no son preferidos no por lo que ello pudiera suponer en el logro de bienestar del sujeto sino por el hecho de que no lo son como agente. Ante esta limitación teorética de los funcionamientos, Sen se escuda en los funcionamientos refinados. Si la definición de funcionamiento primitivo sería "hacer $x$ sin importar qué otras posibilidades alternativas hay" en el funcionamiento refinado se incorpora la importancia de la elección al "escoger hacer x cuando otras alternativas están disponibles" (Sen 1998:290). Tal distinción es insuficiente para superar el escollo que enfrenta el concepto de "metacapacidad", pues la cuestión no solo radicaría en la importancia de las alternativas ni, tan siquiera en la deseabilidad de aquellas -funcionamientos "metarrefinados"- (Sen 2003).

Para enfrentar adecuadamente el asunto debemos incorporar al análisis las "contradicciones potenciales entre los objetivos de agencia”. Si la agencia se define como la facultad inherente del sujeto para juzgar qué cosas tiene valor (Sen 1985a), puede ocurrir que existan situaciones donde aquello que tiene valor suponga una merma para los distintos objetivos de bienestar y de agencia. Esto acontece en aquellas situaciones que valoramos no como deseables sino como necesarias. De tal manera que la evaluación de la actuación no la podríamos comparar con las alternativas presentes sino con aquellas que siendo falsas, en el sentido de contrarias a los hechos, se revelarían verdaderas si estos se manifestaran alternativamente. Para el caso del ejemplo anterior, la libertad de agencia del sujeto que decide permanecer en el lugar del conflicto frente al de la huida comporta un riesgo vital, y por tanto, un perjuicio apreciable en sus objetivos bienestar y de agencia. Elige quedarse, pero preferi- 
ría no tener que hacerlo. La actuación como agente, es decir, permanecer, supone un riesgo considerable sobre sus acciones potenciales como agente. Actuar libremente en este caso sería contraproducente pero no por el hecho de que el individuo así libremente lo decide, sino porque la decisión tomada imposibilitaría cualquier acción futura de agencia.

De nuevo el enfoque seniano intenta superar esta dificultad por medio del concepto de "metapreferencia", esto es, las preferencias de las preferencias (Sen 2002). Argumenta que "la libertad para escoger qué preferencias desarrollar es un aspecto importante de la libertad de agencia" (1999:78); en otras palabras, hubiera preferido preferir aquello que no prefiero. Sin embargo, el caso del ejemplo es diferente ya que aquello que prefiere el sujeto no viene determinado por la libertad frente a lo que desearía preferir sino de circunstancias que se le presentan irreversiblemente. De manera que mientras exista un conflicto armado elegirá la opción de quedarse, pues cambiar de preferencias mermaría sus objetivos de agencia -luchar contra una agresión externa es además un objetivo de agencia deseable si eres patriota-. Por consiguiente, la opción óptima para el sujeto sería la de la ausencia de conflicto.

Tras el análisis, gana relevancia la incorporación analítica del concepto de "metacapacidad", habilitado para identificar aquellos conflictos extremos entre objetivos de agencia y bienestar. De forma que, si ello no fuera posible, la libertad se contrapondría en sí misma a lo pretendido por la ética seniana, resultando normativamente intolerable y fácticamente impracticable (Deneulin y McGregor 2010). A partir de este concepto se deduce cómo la posibilidad para actuar desde objetivos de agencia y bienestar exige que el acto mismo de elección no suponga para sí su negación a posteriori. De este conflicto se produce automáticamente una restricción operativa de la libertad real tanto en sus objetivos de bienestar y de agencia, allende el propio acto libre. Imaginemos un padre de familia que tiene que elegir entre la vida de uno de sus dos hijos de tal forma que cualquier tipo de elección terminara con la trágica muerte de uno de ellos. Aunque como agente no encuentra ningún tipo de restricción para elegir -recordemos que la normatividad en el enfoque seniano no deriva del logro en sí sino de la libertad con el que se alcanza-, el hecho de tener que hacerlo en relación a la vida de uno de sus hijos le supondrá irremediablemente una pérdida de bienestar que lo incapacitará como agente -se vería colapsado ante una situación que le supera-. Este aspecto nos coloca nuevamente en las conclusiones del apartado anterior. Nos encontramos ante logros cuya imposibilidad empírica nos es dada nuevamente desde un ejercicio positivo de libertad (ausencia de normatividad). Su inoperatividad descansa en la incapacidad del agente para valorar las consecuencias -en términos de funcionamientosderivado de aquello por lo que se siente o no tenta- do a elegir. En este sentido, el hecho de presentarse alternativas posibles de elección -la posibilidad de poder elegir que un hijo viva-sería insuficiente para asumirla normativamente como un acto libre.

De todo lo argüido derivamos lo siguiente; y es que, la libertad seniana ("de oportunidad"), a la vista de nuestro análisis, no puede fundamentarse exclusivamente en la mera posibilidad formal de elección. Ser libre para dejar de ser libre podría ser una referencia aceptable de autonomía si la voluntad del sujeto antecede a los acontecimientos. No obstante, cuando son los fenómenos externos aquellos que condicionan prominentemente el grado de determinación moral del logro -ver ejemplo anterior-, actuar libremente supone un ataque contra la libertad misma. Todo esto nos coloca en la frontera de la libertad seniana, donde cualquier superación de tal límite, en un sentido asintótico, generará espontáneamente un estado contraproducente de no-libertad. La libertad solo podrá determinarse fácticamente como principio de justicia si es capaz de someterse a otros presupuestos de acción moral de alcance mayor, donde el grado normativo y positivo de libertad no coincida necesariamente. La libertad como agencia y bienestar debe sostenerse mientras que actuar como agente no repercuta negativamente en su futuro devenir.

\section{Conclusiones}

A lo largo de este artículo se ha pretendido corregir, por medio de la incorporación de una serie inédita de conceptos, el restringido marco de libertad ("de oportunidad") que Sen utiliza a lo largo del enfoque de las capacidades. Y es que, desde la mera asimilación de la libertad "de oportunidad" como variable focal, se hace fáctica y normativamente insostenible "incrementar las oportunidades reales de los individuos para elegir qué tipo de vida desean vivir" (Sen 1984; 1999). El individualismo ético desde el que se suscribe el enfoque provoca que la base informacional quede restringida a los estados individuales, dejando al margen aquellos otros sociales que prominentemente condicionan al modelo. De ello hemos examinado cómo tales estados juegan, desde la libertad "de procesos" en su dimensión social (entorno social), un papel determinante a la hora de dotar de operatividad y normatividad al enfoque.

En primer lugar, reconocimos cómo, a pesar de que Sen hace una nítida distinción analítica entre funcionamientos y capacidades (Sen 1996; 1997; 1999), asemejar la libertad "de oportunidad" a la real supone en términos fácticos un abandono de aquellos otros fenómenos que la determinan, restringiendo operativamente el concepto de capacidad al de mero funcionamiento. De tal impedimento derivamos igualmente cómo el enfoque se encuentra incapacitado para hacer de los logros no solo resultado de funcionamientos específicos sino generadores mismos de nuevos funcionamientos. 
Desde esta interpretación activa del entorno social pudimos examinar cómo las distintas formas en las que se articula afectan al contenido definitivo de las libertades reales. Con la incorporación de las "capacidades sociales" expresamos cómo el entorno social se revela no solo como influencia directa sobre la libertad "de oportunidad" a la hora de expandir los funcionamientos individuales, sino que es en sí mismo un elemento capacitador, determinando la libertad efectiva del sujeto. Las "contracapacidades" nos permitieron ilustrar el aspecto negativo o contracapacitador que el entorno social atesora. Empero, si la libertad para funcionar (capability) nunca se consume absolutamente, la contracapacidad será funcionar necesariamente en términos negativos. Términos que nos obliga a incorporar el condicionante ético en los funcionamientos distinguiendo entre una libertad para funcionar exclusivamente (positivamente) y otra para hacerlo normativamente. El tercero de los conceptos nos llevó a un aspecto más profundo en la determinación de los funcionamientos. Con el concepto de "metacapacidad" ilustramos cómo el entorno social puede ser vórtice de conflictos entre objetivos potenciales de agencia y bienestar de forma que funcionar de un modo determinado hipotecara la libertad para poder hacerlo de cualquier otra manera. Desde todo este andamiaje teórico hemos escenificado un acercamiento analítico al enfoque de las capacidades que nos ha permitido perfeccionar el marco categorial seniano de capacidades individuales por medio de la incorporación de una serie de conceptos que asociados a la dimensión social de la libertad "de procesos" nos aproximan a un estado absoluto de capacidad.

\section{Notas}

1. En este trabajo no se abordará la cuestión de las capacidades absolutas como ente analítico autónomo y sí aquellos fenómenos que lo determinan. Su inclusión en el apartado introductorio de este trabajo es una manera aunque gruesa de dotar de unidad de análisis a los distintos elementos que examinaremos a lo largo del artículo. Su análisis como concepto general queda suspendido a próximos trabajos.

2. Intentando contrarrestar esta tendencia, investigadores como Robeyns $(2005,2009)$ se han apuntado a una especie de defensa seniana donde la libertad de procesos quede asumida desde el propio quehacer de la libertad "de oportunidad", de forma que el principio de acción que se revela de esta segunda fuera condición suficiente de la primera. Con objeto de persuadir sobre tal empresa se ve forzado a realizar una distinción terminológica entre individualismo ético y metodológico, que confunde más que aclara. Para Robeyns es compatible el primero con una epistemología que rechace al segundo. Sería imposible aceptar la libertad individual si no se considerara aquellos aspectos que finalmente termina afectándola -comenta el autor-. Desde esta defensa se recupera el problema de fondo de la teoría de las capacidades y la lógica de este trabajo. Aunque someter la libertad "de procesos" a la libertad de oportunidades exige aceptar lo sostenido por Robeyns, lo sería tan solo sometiendo la realidad social a la esfera restringida de la experiencia individual.

3. Las referencias al concepto de bien o bienes siempre lo serán desde el sentido más amplio dado por Sen donde se incluyan también los estados personales (Sen 1984).

4. Aunque habitualmente capacidad y funcionamiento son indistintamente utilizados por Sen -particularmente cuando son referidos a un funcionar determinado-, para esta específica cuestión su distinción es meritoria, pues el punto de énfasis coincide con el de su distinción. Estar capacitado responde a la libertad para poder funcionar de esa manera o de cualquier otra dentro de los funcionamientos posibles.

5. La relevancia del entorno social como determinante en la formación de las libertades para poder funcionar (capabilities) no es recogida de forma inédita en este trabajo. Aunque más superficialmente ya algunos autores han llegado a intuir la importancia de asumir el entorno social como condición necesaria para poder funcionar
(Nussbaum 2002 y 2012; Deneulin 2014).Empero este énfasis sobre el entorno no lo es en tanto que generador de capacidades en sí, y sí a partir de la interacción de la libertad "de procesos" con la libertad "de oportunidad". Tomando el concepto de "capacidades combinadas" desarrollado por Nussbaum (2002) "capacidades interiores más las condiciones externas convenientes para el ejercicio de una función", observamos cómo la libertad, para poder funcionar, queda necesariamente constreñida a funcionar o no de una manera particular pero no de hacerlo desde otras muchas. Para aquellas otras formas se hace necesario recuperar aquellos fenómenos que determinan la posibilidad para funcionar pero cuya génesis deriva del propio entorno social y que Nussbaum nunca llega a rescatar completamente. Es precisamente aquí donde el concepto de "capacidades sociales" gana toda su relevancia.

6. Amartya Sen (1999) apuesta por un concepto de bienestar amplio (eudamonia) que supere el reduccionismo de entender como bienestar la mera preferencia personal. Los individuos no siempre buscan su bienestar sino que en ocasiones anteponen otros intereses derivados de perseguir objetivos sociales desinteresadamente. Para ello hace un desdoblamiento del bienestar amplio que incorpore los objetivos de bienestar personal, entendido como aquellos logros cuya utilidad deriva de la mera satisfacción personal, y los objetivos de agencia (agency) donde los logros vengan dados de la facultad del individuo (como agente) para vivir una vida considerada como deseable. Empero, el concepto de bienestar amplio no queda restringido a lo meramente logrado ya sea en cuanto a logros de bienestar o agencia. El grado de libertad con los que estos son alcanzados (libertad de bienestar y libertad de agencia) será crucial a la hora de valorar la calidad de vida.

7. Lo normativamente deseable de la libertad vendrá definido grosso modo en términos negativos como lo opuesto a una ética basada en el mero incremento positivo de las libertades para funcionar. Así, contrario a Nussbaum (2002) la cual defiende un índice de funciones básicas taxonomizadas, el economista indio es contrario al establecimiento de una lista de capacidades a riesgo de someter la libertad individual a una especie de "esencialismo internalista" que impida la diversidad que anida en el individuo (Sen 2004; 2009). En perjui- 
cio de esto, somete el carácter ético de la libertad a un proceso auto-reflexivo sin referencia exógena normativa. Con ello, un incremento asintótico en las posibilidades con las que funcionar (libertad como capacidad) se revela normativamente suficiente para sí, a riesgo de invisibilizar como realmente ocurre, aquellos fenómenos que trascendiendo la variable focal seniana determinan el juicio sobre una buena vida (¿qué libertad como capacidad?). Es la misma Nussbaum la que en

\section{RefERENCIAS Bibliográficas}

Agee, M. y T. Crocker. 2012. "Operationalizing the Capability Approach to Assessing Well-being". Journal of SocioEconomics 46: 80-86. http://dx.doi.org/10.1016/j.socec.2013.07.003

Berlin, I. 2002. Sobre la libertad. Madrid: Alianza Editorial.

Cejudo, R. 2007. "Capacidades y libertad. Una aproximación a la teoría de Amartya Sen". Revista Internacional de Sociología 65:9-22.

Cohen, G. 1996. "¿Igualdad de qué? Sobre el bienestar, los bienes y las capacidades". Pp. 222-243 en La calidad de vida, compilado por M. Nussbaum. México: Fondo de Cultura Económica.

Cortina, A. 2002. Por una ética del consumo. Madrid: Taurus.

Deneulin, S. 2006. Transforming Unjust Structures the Capability Approach. The Netherlands: Springer. http:// dx.doi.org/10.1007/1-4020-4432-1 PMid:17409893

Deneulin, S. 2011. "Development and the Limits of Amartya Sen's The Idea of Justice". ThirdWorldQuarterly 32:78797. http://dx.doi.org/10.1080/01436597.2011.567008

Deneulin, S. 2014. Well-being, Justice and Development Ethics. London: Routledge.

Deneulin, S. y McGregor. 2010. "The Capability Approach and the Politics of a Social Conception of Well-being". European Journal of Social Theory 13:501-19. http:// dx.doi.org/10.1177/1368431010382762

Giri, A. 2000. "Rethinking Human Well-being: a dialogue with Amartya Sen". Journal of International Development 12:1003-1018. http://dx.doi. org/10.1002/1099-1328(200010)12:7<1003::AIDJID698>3.0.CO;2-U / http://dx.doi.org/10.1002/10991328(200010)12:7<1003::AID-JID698>3.3.CO;2-L

Leßmann, O. 2012. "Applying the Capability Approach Empirically: An Overview with Special Attention to Labor". Management Review 23:99-118.

Nozick, R. 1988. Anarquía, estado y utopía. México: Fondo de Cultura Económica.

Nussbaum, M. 2002. Las mujeres y el desarrollo humano: el enfoque de las capacidades. Barcelona: Herder.

Nussbaum, M. 2011. "Capabilities, Entitlements, Rights: Supplementation and Critique". Journal of Human Development and Capabilities 12:23-37. http://dx.doi.org/10. 1080/19452829.2011.541731

Otaga, S. 2003. "Human Security and State Security". New York: Comisión de Seguridad Humana, Human Security Now.

Rawls, J. 1971. Una teoría de la justicia. Boston: Harvard University Press. PMCid:PMC2609853

Robeyns, I. 2005. "The Capability Approach: A Theoretical Survey". Journal of Human Development 6:25-36. http://dx.doi.org/10.1080/146498805200034266

Robeyns, I. 2009. "Equity and Justice". Pp. 101-120 en An Introduction to the Human Development and Capability Approach: Freedom and Agency, editado por S. Deneulin y L. Shahani. London: Earthscan. PMCid:PMC2790901 defensa de una lista mínima de capacidades sostiene que "poseer estas capacidades es propio del ser humano", evitando con ello que lo positivo y normativo de la libertad coincidan necesariamente. Esto desvela cómo el carácter normativo de un juico, para este caso el seniano, nunca se consume completamente en las bases de su propio discurso y sí en la posibilidad de someterse a presupuestos normativos de alcance mayor (qué libertad frente a cualquier libertad).

Sen, A., R. Muellbauer, K. Kanbur y K. Hart. 1987. "The Standard of Living". The Tanner Lectures in Human Values, editado por G. Hawthorn. Cambridge: Cambridge University Press. http://dx.doi.org/10.1017/ cbo9780511570742

Sen, A. 1973b. On Economic Inequality. Oxford: Clarendon Press. http://dx.doi.org/10.1093/0198281935.001.0001

Sen, A. 1982. "Liberty as Control: An Appraisal". Journal of Midwest Studies in Philosophy 7:207-221. http://dx.doi. org/10.1111/j.1475-4975.1982.tb00092.x

Sen, A. 1984. Goods and People. Cambridge, Mas.: MIT press.

Sen, A. 1985a. "Well-being, Agency and Freedom". Journal of Philosophy 82:169-221. http://dx.doi.org/10.2307/2026184

Sen, A.1985b. Commodities and Capabilities. Amsterdam: North-Holland.

Sen, A. 1998. "Freedom and Choice: Concept and Content". European Economic Review 32:269-294. http://dx.doi. org/10.1016/0014-2921(88)90173-0

Sen, A. 1999. Development as Freedom. New York: Oxford University Press.

Sen, A. 2000. Nuevo examen de la desigualdad. Madrid: Alianza.

Sen, A. 2002b. Rationality and Freedom. Cambridge: Belnap Press of Harvard University Press.

Sen, A. 2003. Sobre Ética y Economía. Madrid: Alianza Editorial.

Sen, A. 2004. "Capabilities, List and Public Reason: Continuing the Conversation". Feminist Economics 10:77-80. http://dx.doi.org/10.1080/1354570042000315163

Sen, A. 2006. "What do we Want from a Theory of Justice?" Journal of Philosophy 103:215-238. http://dx.doi. org/10.5840/jphil2006103517

Sen, A. 2009. The Idea of Justice. London: Penguin Group.

Sen, A. 2013. "The Ends and Means of Sustainability". Journal of Human Development and Capabilities 14:6-20. http://dx.doi.org/10.1080/19452829.2012.747492

Streeten, P. 1984. "Basic Needs: some Unsettled Questions". World Development 12:973-978. http://dx.doi. org/10.1016/0305-750X(84)90054-8

Steward, F. 2013. "Nussbaum on the Capabilities Approach". Journal of Human Development and Capabilities 14:156-60. http://dx.doi.org/10.1080/19452829.2013. 762175

Uribe López, M. 2009. "Metas blandas y metas duras en el enfoque de Desarrollo Humano". Revista de la Universidad Bolivariana 8:181-193. http://dx.doi.org/10.4067/ s0718-65682009000200009

Van Parijs, P. 1996. Libertad real para todos. Qué puede justificar el capitalismo (si hay algo que pueda hacerlo). Barcelona: Paidós.

Van Staveren, I. 2001. The Values of Economics: An Aristotelian Perspective. London: Routledge. http://dx.doi. org/10.4324/9780203164037 
MANUEL A. JIMÉNEZ-CASTILLO. Doctor en Ciencias Económicas por la Universidad de Sevilla. Magister en Desarrollo Económico por la London School of Economics and Political Science. Profesor-Investigador en El Colegio de la Frontera Norte. Ha trabajado como profesor en desarrollo económico y teoría micro-financiera en varias universidades del sudeste asiático. Autor de artículos en revistas nacionales e internacionales. 